\title{
La tecnología blockchain una alternativa para revolucionar el sector turístico cubano
}

Blockchain technology, an alternative to revolutionize the Cuban tourism

sector

1 María Teresa García Andraca

Universidad de La Habana, Facultad de Turismo, Carrera de Licenciatura en Turismo,

Cuba.

tg618039@gmail.com

2 Yucert Hernández Calderón

Universidad de La Habana, Facultad de Turismo, Carrera de Licenciatura en Turismo,

Cuba.

yucert99@gmail.com

3 Yasser Vázquez Alfonso

Universidad de La Habana, Facultad de Turismo, Departamento de Turismo. La Habana,

Cuba

yalfos1@gmail.com

4 Luis Efrain Velastegui Lopez $\quad$ (iD) https://orcid.org0000-0002-7353-5853

Universidad Técnica de Babahoyo, Babahoyo, Ecuador

evelasteguil@utb.edu.ec

Artículo de Investigación Científica y Tecnológica

Enviado: 05/12/2021

Revisado: $17 / 12 / 2021$

Aceptado: 27/01/2022

Publicado:26/02/2022

DOI: https://doi.org/10.33262/exploradordigital.v6i1.2078

Cítese: García Andraca, M. T., Hernández Calderón, Y., Vázquez Alfonso, Y., \& Velastegui Lopez, L. E. (2022). La tecnología blockchain una alternativa para revolucionar el sector turístico cubano. Explorador Digital, 6(1), 31-50. https://doi.org/10.33262/exploradordigital.v6i1.2078

EXPLORADOR DIGITAL, es una Revista electrónica, Trimestral, que se publicará en soporte electrónico tiene como misión contribuir a la formación de profesionales competentes con visión humanística y crítica que sean capaces de exponer sus resultados investigativos y científicos en la misma medida que se promueva mediante su intervención cambios positivos en la sociedad. https://exploradordigital.org

La revista es editada por la Editorial Ciencia Digital (Editorial de prestigio registrada en la Cámara Ecuatoriana de Libro con No de Afiliación 663) www.celibro.org.ec 


Palabras
claves:
blockchain,
turismo, Cuba,
tecnología.

Keywords:

blockchain, tourism, Cuba, technology.

\section{Resumen}

Cuba se encuentra actualmente enfrascada en el proceso de informatización de la sociedad, que incluye apropiarse de las ventajas y la cultura de los avances tecnológicos y de las info comunicaciones. Un paso importante en esta dirección podría ser el estudio y conocimiento profundo de la tecnología blockchain. La tecnología blockchain se presenta como una revolución que soluciona los problemas de vulnerabilidad que ha venido presentando la Internet, así como una posibilidad de aplicación frente a los problemas de seguridad informática indescifrable a terceros. Esta tecnología ha comenzado a impactar en diversos ámbitos más allá de la informática. A pesar de esto, los estudios existentes sobre el tema son aún insuficientes, especialmente en Cuba. Por tal motivo, se hace necesaria la investigación sobre esta tecnología disruptiva y sus aplicaciones en el sector turístico. En la presente investigación se realiza una sistematización del tema. En un primer momento, se efectúa un acercamiento al fenómeno; estableciendo su historia, principales definiciones, ventajas, desventajas y tipos de red; dado que la información sobre la evolución y características del blockchain es aún poco conocida y difundida. En un segundo capítulo se efectúa un análisis sobre sus posibles aplicaciones en el sector turístico, para finalmente desde la coyuntura económica de Cuba, evaluar sus potencialidades de uso. La presente investigación pretende ser un primer acercamiento al tema de manera tal que se difundan los potenciales usos del blockchain en el sector turístico cubano y se abran nuevas investigaciones en este sentido.

\section{Abstract}

Cuba is currently immersed in the process of informatization of society, which includes the appropriation of the advantages and culture of technological advances and info communications. A crucial step in this direction could be the study and deep knowledge of blockchain technology. Blockchain technology is presented as a revolution that solves the problems of vulnerability that the Internet has been presenting, as well as a possibility of application against the problems of computer security undecipherable to third parties. This technology has begun to have an impact in various fields beyond IT. Despite this, existing studies on the subject are still insufficient, especially in Cuba. Therefore, it is necessary to 
investigate this disruptive technology and its applications in the tourism sector. In this research a systematization of the subject is conducted. At first, an approach to the phenomenon is made; establishing its history, main definitions, advantages, disadvantages and types of networks; given that the information on the evolution and characteristics of the blockchain is still little known and disseminated. In a second chapter, its applications in the tourism sector are analyzed and, finally, based on Cuba's economic situation, its use is evaluated. This research aims to be a first approach to the topic to disseminate the possible uses of blockchain in the Cuban tourism sector and to open new research on the subject.

\section{Introducción}

Las diferentes entidades dedicadas a un sector con propósitos de ofrecer un determinado servicio están expuestas a continuos cambios con el fin de lograr una adecuada atracción y fidelización de sus clientes. La clientela es la máxima autoridad en estas empresas. Son estos los que, mediante su constante asistencia o no al producto/servicio, pueden hacer germinar o extinguir una determinada entidad.

El turismo es uno de estos sectores por lo que está sujeto a elevados procesos donde la verificación de la identidad e información de los usuarios es un paso indispensable y depende en lo absoluto del modelo de negocio. Por tanto, existe un inmenso "libro de registro" a través del que distintas entidades y personas pueden intercambiar información y valor.

El progresivo uso tecnológico en empresas turísticas ha implementado el vínculo entidadcliente, siendo de gran importancia para almacenar la información. Estas entidades han ido modificando sus activos tecnológicos para su uso en las actividades empresariales, destacándose como vía modal durante los últimos años el desarrollo del comercio electrónico. Esta lejana relación requiere mayor seguridad y transparencia empresarial hacia el cliente, el cual espera obtener una clara satisfacción en la entidad a visitar.

El comercio electrónico (E-commerce) ha llegado a depender mayoritariamente de terceras instituciones financieras, confiables en el proceso de los pagos electrónicos. A pesar de que el sistema funciona suficientemente bien en la mayor parte de las transacciones, sufre la debilidad inherente al modelo basado en confianza. La aplicación de la tecnología a la automatización de procesos de negocio y flujos de trabajo, aumentando el grado de satisfacción y, mediante una reducción de costes, la calidad de los productos (Coin ATM Radar, 2021). 
El sistema actual de transferencia de valor a través de medios digitales se basa en la confianza que los usuarios depositan en determinada entidad, la cual adquiere cierta autoridad y ejerce un papel de intermediario ofreciendo privacidad y seguridad. Esto no es un hecho aislado de la economía digital. Durante años, los seres humanos han utilizado billetes impresos en papel, cuyo valor material no va acorde al importe que representan. Sin embargo, se confía en que tanto los demás usuarios como las entidades bancarias van a darle a ese papel la misma validez. Esta misma presunción se ve reflejada en cada una de las transferencias de información y/o valor que se realizan a través de internet cada día: desde los bancos que aseguran que el dinero se transfiere a la persona deseada, pasando por los certificados digitales de gobiernos para determinar que un documento es fidedigno, hasta con el mero hecho de mandar un mensaje a través de mensajería móvil y que el proveedor asegure que ha sido entregado. No obstante, la tecnología blockchain se presenta como una tecnología disruptiva que surge como una solución a los graves problemas de vulnerabilidad que ha venido presentando la Internet, así como una posibilidad de aplicación frente a los problemas de ciberseguridad indescifrable a terceros.

El término "tecnología disruptiva" fue llevado a cabo a mediados de los 90's ya que se comenzó a utilizar para todas aquellas tecnologías que, además de permitir la innovación en procesos (innovación continua) o crear nuevos productos/servicios (Innovación incremental), tenían la capacidad de transformar los modelos de negocio. Precisamente, esta última transformación de modelos de negocio se calificó como innovación disruptiva, y las tecnologías que permitían dicha transformación fueron calificadas como Tecnologías Disruptivas.

La naturaleza cada vez más hostil del ámbito económico-financiero en que se debe desenvolver Cuba, provocan mirar al blockchain y las criptomonedas desde la realidad cubana, para determinar si pudieran ser una alternativa factible y viable para Cuba. El blockchain y el turismo en Cuba tienen el potencial de transformarse en una mezcla muy ventajosa, ya que esta tecnología puede aportar seguridad y transparencia en ciertos aspectos. El blockchain puede hacer que las transferencias y almacenamiento de informaciones sean más fáciles y seguros, ya que la responsabilidad se comparte por toda una red. Lo mismo ocurre con los pagos en el extranjero, lo que aumenta el nivel de confianza entre los interesados.

En ese sentido, la investigación tiene como objetivo general exponer las principales características, potencialidades y limitaciones de la implementación de tecnología blockchain para su aplicación en instalaciones turísticas cubanas. 


\section{Metodología}

La investigación fue realizada en la etapa correspondiente al confinamiento generado por la COVID-19. En cuanto a los métodos teóricos utilizados se encuentran el hipotéticodeductivo empleado para determinar la hipótesis de la investigación; y el histórico lógico que posibilitó entender las características de blockchain a lo largo de su historia. Los procedimientos empleados fueron el análisis y síntesis que permitió conocer profundamente las realidades, así como simplificar las revisiones bibliográficas; y la inducción- deducción que se empleó para obtener conclusiones, partiendo de lo general hacia lo particular. Dentro de los métodos empíricos se pueden mencionar la observación externa, abierta y directa utilizada para determinar las características existentes en las instalaciones que faciliten el desarrollo de nuevas tecnologías. Todos estos métodos y procedimientos permitieron procesar la información obtenida y sintetizarla para la consecución de los objetivos propuestos.

\section{Resultados}

\section{Historia y definición de blockchain}

El blockchain, o cadena de bloques (como se conoce en español), es proclamado en la actualidad como uno de los inventos que ha revolucionado el mundo. Desde las bases de datos, la economía y las formas de hacer transacciones en criptomonedas, más allá del dinero tradicional.

La novedosa herramienta económica blockchain pudiera estar inspirada en los primeros registros contables del año 3200 A.C., debido a que la humanidad los conoce como los primeros datos ordenados de forma sistemática. Luego en 1494 se crea el primer sistema de contabilidad de doble entrada.

La historia del blockchain, ya en la era actual, se remonta a más de 40 años de estudios y pruebas. Estas hicieron posible que hoy en un mundo globalizado, existan transferencias a través de esta cadena de bloques; además de la trazabilidad de productos y servicios (López, 2019).

Allende (2018), afirma que la primera publicación sobre esta tecnología fue en 1991. La idea consistía en tener un registro digital de archivos ordenados cronológicamente, permitiendo conocer con exactitud su autoría y fecha de creación. Seis años más tarde, Adam Back creó Hashcash, un sistema monetario alternativo que usaba lo que se consideraría, hoy día, una primera prueba del sistema que popularizó el bitcoin (BTC). Ya en 1998 en los sistemas B-Money y Bit Gold de WeiDai y Nick Szabo se introdujo la noción de capacidades digitales distribuidas para la gestión de criptomonedas (Iglesias, 2018). 
La puesta en escena del primer blockchain fue con la llegada de BTC, sobre el 2008-2009 (Berenguer, 2017), a manos de Satoshi Nakamoto. El bitcoin, como criptomoneda, fue utilizado para proveer un método de pago electrónico que no necesita supervisión y elude el control de las instituciones financieras.

El 22 de mayo de 2010 se produjo la primera transacción real con BTC (el pago de dos pizzas por un valor de 10.000 BTC) (Iglesias, 2018). Esta criptomoneda fue la inspiración para que posteriormente otras iniciativas aparecieran como parte de plataformas abiertas para el intercambio de transacciones, tales como Ripple, en 2012 y Etherum, en 2015. En la actualidad existen más de 8.400 criptomonedas (Castro, 2021), donde el BTC sigue siendo el líder indiscutible con un valor equivalente que ha superado los 60 mil USD en el mes de abril del presente año (El Economista, 2021).

\section{Definiciones de blockchain}

Blockchain puede definirse según varios autores como:

* una tecnología que puede -para ciertas aplicaciones o sectores- eliminar intermediarios, también llamados terceros de confianza (Coin ATM Radar, 2021).

* una base de datos compartida que funciona como un libro para el registro de operaciones de compraventa o cualquier otra transacción (Yahari, 2019).

una nube descentralizada y base de datos distribuida de código abierto que vincula todos los registros por consenso (Kwok \& Koh, 2018).

* una tecnología que forma parte del ámbito de las denominadas tecnologías de registro distribuido o DLT (Distributed Ledger Technology). Permite gestionar datos, ordenes, transacciones, activos y tokens, mediante un sistema de registro descentralizado que se anota en bloques de información los cuales se encadenan secuencialmente creando una cadena de registros inmutable e inalterable (Palomo-Zurdo, 2018).

A modo de conclusión, se puede considerar blockchain a "una tecnología descentralizada que consiste en un número de transacciones almacenadas y organizadas en bloques, con códigos alfanuméricos inmutables e indestructibles, en la que se pueden registrar operaciones de compraventa provocando la eliminación de intermediarios durante el proceso".

\section{Tipos de blockchain}

Esta tecnología ha generado la popularidad en todos los sectores empresariales, surgiendo un tipo de mercado diferente, el BaaS (Blockchain as a Service). Los modelos de BaaS han logrado que el desarrollo tecnológico sea más simple y seguro para las organizaciones cuyas competencias centrales se encuentran alejadas del sector de la tecnología de la 
información. El BaaS es un modelo único que permite a los consumidores utilizar los servicios basados en la nube para desarrollar, usar y alojar sus aplicaciones, funciones y contratos inteligentes (Vector ITC, 2018). El mismo autor plantea que, dentro de la nueva tecnología, existen tres tipos de blockchain: público, privado e híbrido:

La red de blockchain pública posee códigos abiertos y permite a cualquier persona participar como miembro, usuario, minero, etc. Es una red diseñada para estar completamente descentralizada, sin que ningún individuo o entidad controle qué transacciones se registran o el orden en que son procesadas. Las transacciones son totalmente transparentes.

\section{Figura 1}

Esquematización de blockchain público
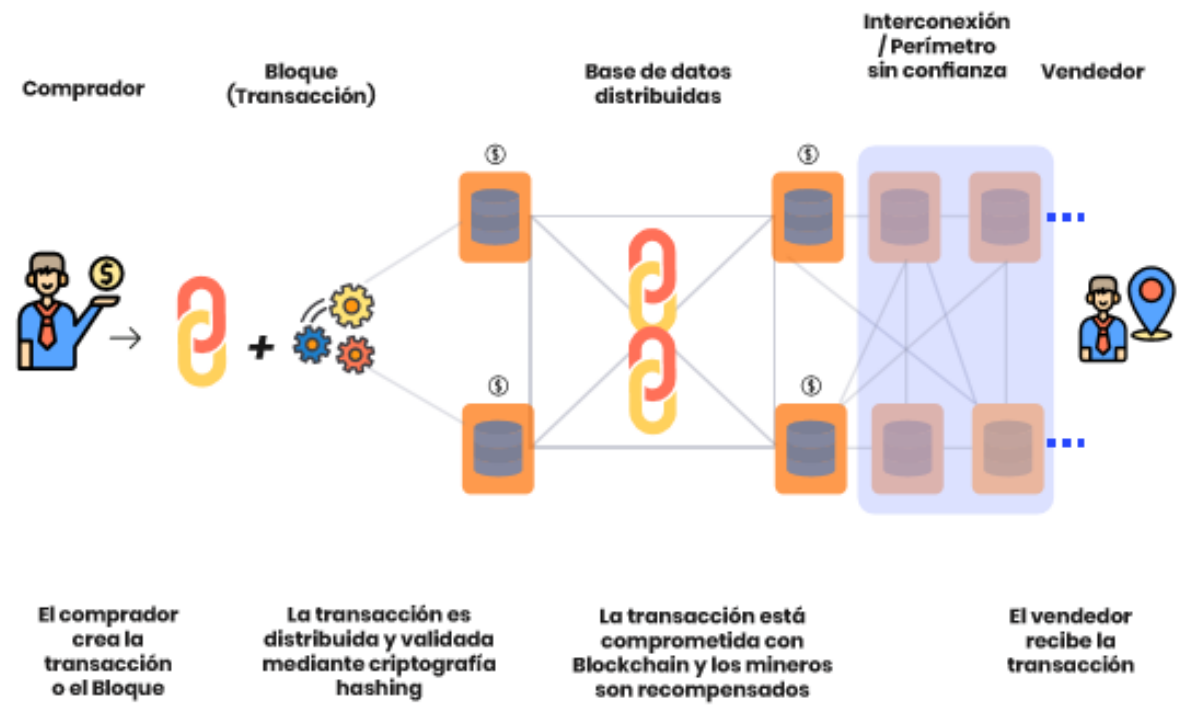

El vendedor

recibela

transacción hashing son recompensados

Fuente: Vector ITC (2018)

Más centralizadas que las redes de blockchain públicas, las cadenas de bloques privadas pueden ser fácilmente distinguidas de las demás. Son valiosas para las empresas que desean colaborar y compartir datos, pero no quieren que sus datos comerciales confidenciales sean visibles en una cadena de bloques pública. Las transacciones son privadas y solo están disponibles para los participantes del ecosistema que tienen permiso para unirse a la red. 


\section{Figura 2}

Esquematización de blockchain privado

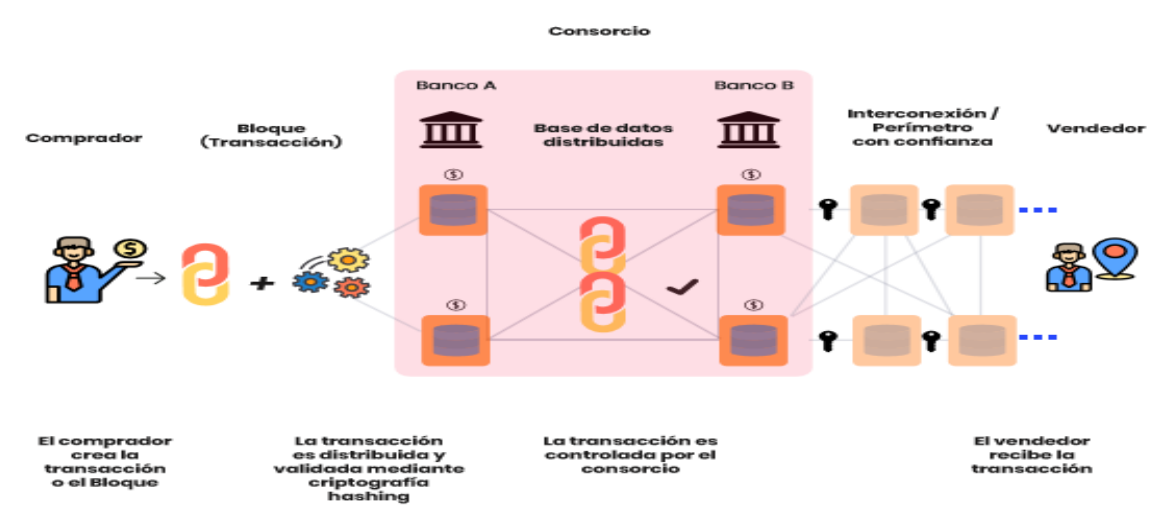

Fuente: Vector ITC (2018)

La cadena de bloques híbrida es una combinación de los extremos de blockchain públicos y privados, aprovechando las características de ambos. Los miembros de la red o las entidades dominantes pueden decidir qué transacciones pueden seguir siendo públicas y cuáles estarán abiertas a un grupo más pequeño de miembros. Este tipo de blockchain puede ayudar a construir herramientas a nivel empresarial de la tecnología de código abierto y su sólida seguridad permite su uso en pagos transfronterizos confiables para financiación y comercio.

\section{Ventajas y desventajas de la tecnología blockchain}

Con el blockchain actuando como un mecanismo con nodos sincronizados, las transacciones se pueden verificar y procesar de manera independiente. Esta función provoca que la confianza de los usuarios aumente al igual que sus transacciones (ver figura3), existiendo en el último mes del año 2017 más de diez mil millones de transacciones en BTC (Fernández, 2020). Su creación trajo a su par numerosas ventajas en diferentes industrias, facilitando una seguridad incrementada en sistemas trustless (los involucrados no necesitan conocerse o confiar entre sí o en un tercero para que el sistema funcione) (Equipo Cazoo, 2021).

Pero su entorno también comporta ciertas desventajas.

Ventajas:

$\checkmark$ Transacciones ejecutadas como ordena el protocolo con costes muy bajos para el intercambio de activos.

$\checkmark$ Es un método seguro donde las transacciones son inmutables. 


\section{EDDigital}

Transparente, pues los datos están integrados en la red como un todo, lo que se puede ver públicamente.

$\checkmark$ Los usuarios tienen el control de toda su información y transacción.

$\checkmark$ Datos almacenados en múltiples dispositivos en una red distribuida de nodos con sistema y datos altamente resistentes a fallos técnicos y ataques maliciosos.

$\checkmark$ No presenta un "single point of failure" (punto único de fallo) ya que cada nodo replica y almacena una copia de la base de datos.

Desventajas:

Una vez se han añadido datos a los mismos, resulta muy difícil modificarlos.

$\checkmark$ A pesar de su eficiente seguridad existen algunos tipos de ataques potenciales que pueden ser llevados a cabo contra redes blockchain, aunque nunca se ha producido un ataque del 51\% que haya tenido éxito en la blockchain de BTC (Binance Academy, 2018).

$\checkmark$ Aunque minimiza costos de transacción y tiempo, los altos costes iniciales de capital podrían ser un impedimento.

$\checkmark$ Cada criptomoneda requerirá un sistema de minería y éste a su vez de una gran cantidad de consumo de energía.

$\checkmark$ Representa un cambio completo a una red descentralizada que requiere la aceptación de sus usuarios y operadores.

\section{Figura 3}

Transacciones en BTC desde 2016 hasta marzo de 2020

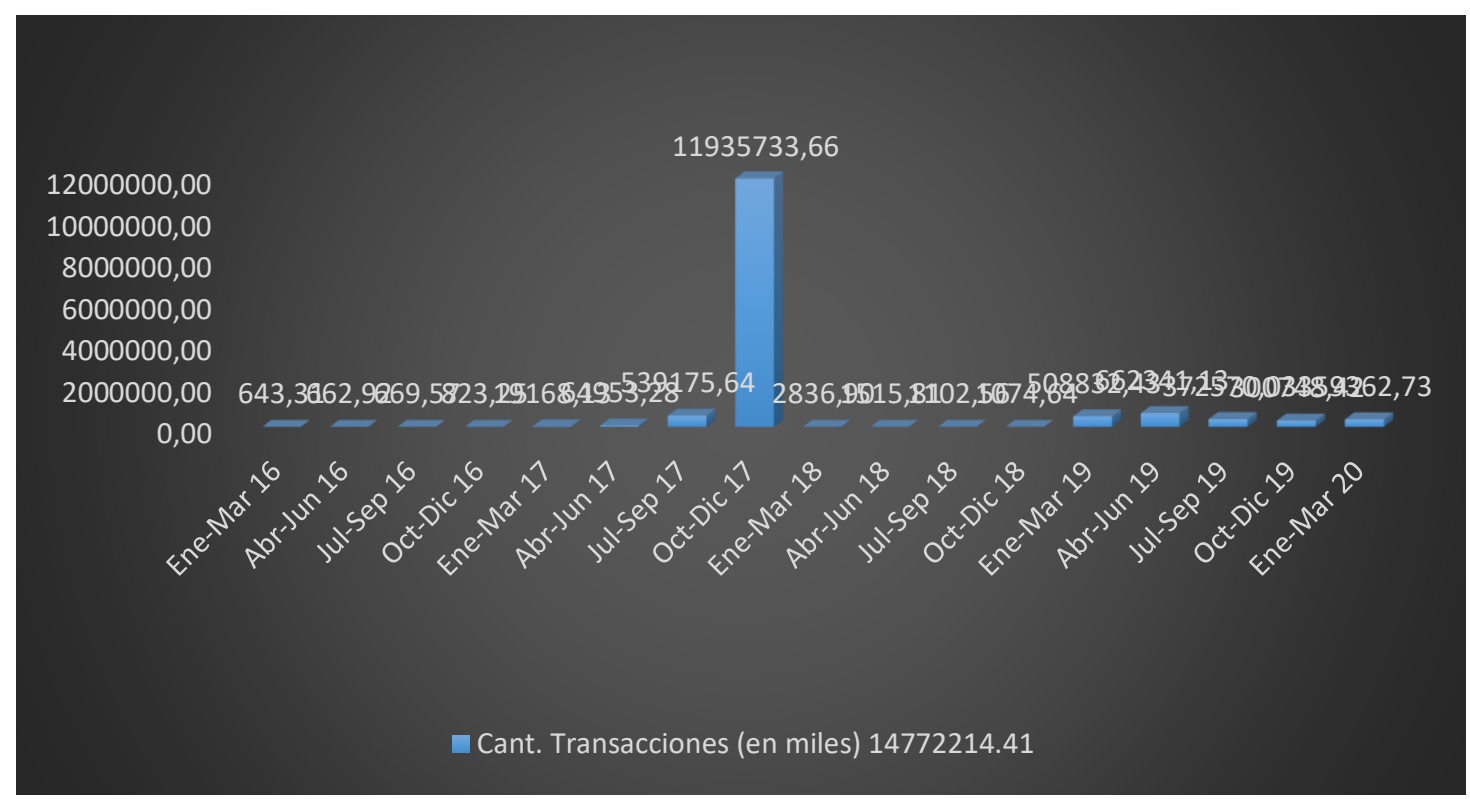




\section{Beneficios que aporta la tecnología blockchain al sector turístico}

La actividad turística, impulsada y apoyada en las tecnologías, es muy susceptible a ser "tokentizada". A raíz de las exigencias de los clientes, cada instalación debe implementar nuevas formas de pago para la venta de productos, creando una sinergia entre los métodos más tradicionales, hasta la aceptación de las nuevas tecnologías para el comercio y la administración de datos.

La tecnología disruptiva blockchain, tiene capacidad de optimizar la eficiencia del flujo de trabajo de cualquier industria., donde una de sus ventajas importantes en el turismo es la identidad única, donde las partes comparten la información de una vía segura. Esta tecnología se encuentra en un punto de partida dentro del turismo, no obstante, se prevé su implementación en diversos ámbitos, aprovechando sus singularidades en la optimización de procesos. Varias empresas vinculadas al turismo ya utilizan esta tecnología, la cual se espera que sea el componente central de muchos negocios para 2025 (Willie, 2019).

\section{Alojamiento}

En primer lugar, en el ámbito de la gestión de ingresos (revenue management), un ejemplo de la aplicación de la cadena de bloques es Locktrip, agencia de viajes online que ha logrado ser la primera plataforma blockchain dentro del sector turístico. El utilizar su propia criptomoneda o token genera una gran ventaja, pues se evita la pérdida de dinero debido al cambio de moneda y además posee las comisiones más bajas del mercado (Willie, 2019).

En segundo lugar, para empresas de alojamiento con más de un emplazamiento de características similares, la blockchain puede ser usada para el inventory management o en español la gestión del inventario. Desde este punto de vista puede ayudar a determinar qué equipamiento se posee, en qué estado está, su localización en tiempo real y si será requerido para ciertos días en particular (Willie, 2019).

El mismo autor expone que otra de las potenciales aplicaciones de la cadena de bloques son los historiales de clientes; lo que favorece el proceso de reubicación de algún huésped, para personalizar la experiencia y ofrecer un mejor servicio en caso de lograr una repetición de la experiencia en una misma cadena hotelera.

\section{Turoperadores y agencias de viajes}

En relación con las agencias de viajes (AAVV) y turoperadores (TTOO) la tecnología blockchain también aporta importantes ventajas ya que existen varias AAVV online basadas en ella. TUI, una de las grandes empresas turísticas, fue la primera AAVV en incluirla (Willie, 2019). La emergente tecnología ofrece un ámbito digital seguro para el 
traspaso de información entre el cliente y el TTOO, ya que la responsabilidad se comparte por toda una red además que brinda la posibilidad de tener esa información accesible en todo momento. Cualquier transacción, compra, itinerario o excursión será fácilmente rastreable en cualquier momento, ya que se creará un nuevo bloque y se añadirá a la cadena.

\section{Industria aérea}

Olvidarse del pasaporte, del DNI o de la tarjeta de embarque en casa al viajar, deja de ser un problema gracias a blockchain y a la biometría, tecnología que verifica aspectos biológicos para reconocer personas. Con estas tecnologías, no solo el viajero podrá olvidar en casa sus documentos, sino que además ahorrará más tiempo de lo habitual. El proceso de identificación de pasajeros pasa a ser rápido y producirá una circulación de pasajeros más fluida.

Otra de las utilidades de blockchain en lo referente a la industria aérea es el rastreo a tiempo real del equipaje una vez el cliente se separe de él en el mostrador de facturación, resultando beneficioso tanto para la aerolínea como para los viajeros.

\section{Sostenibilidad y calidad}

Las innovaciones tecnológicas ofrecen nuevas formas de enfrentar los desafíos medioambientales y gestionar los recursos del planeta. Blockchain permite aunar los esfuerzos globales para lograr el cumplimiento de los Objetivos de Desarrollo Sostenible (ODS), aportando transparencia y trazabilidad.

Varios países han concretado iniciativas para enfrentar de manera conjunta los efectos derivados de la contaminación ambiental. Entre los proyectos existentes se encuentran: Bankia en España que desarrolla un plan blockchain para la reducción de emisiones de gases de efecto invernadero y el Centro Nacional de Energías Renovables que ha desarrollado GreenLedger, una plataforma que usa blockchain para registrar los diferentes eventos de una planta virtual de generación y almacenamiento de energía, donde sus componentes puedan estar ubicados en puntos geográficos muy distantes o incluso pertenecer a distintas compañías.

\section{Comercio, exportaciones y logística}

Las nuevas tecnologías son una puerta hacia la disrupción de los servicios logísticos y del comercio tal como es conocido. Blockchain está haciendo lo propio para el comercio en general. Según Ganne (2018) la Organización Mundial del Comercio plantea tres grandes dimensiones mediante las cuales esta tecnología promete revolucionar el comercio internacional: aumento de la confianza y la transparencia en las cadenas de valor; 
reducción de los costos comerciales y oportunidades para las MIPYMES (pequeñas y medianas empresas) y los pequeños productores de los países en desarrollo.

Cada vez más se está utilizando esta tecnología como sistema de datos en las cadenas de suministros debido a los altos niveles de confianza y la visibilidad que proporciona. De igual modo, blockchain se está implementando para contratos inteligentes que se activan en automático una vez que se ha producido un evento (como entregas finales de productos). Estas propuestas animan a promover su uso en distintos aspectos del comercio, la logística y las exportaciones.

\section{Gastronomía}

Blockchain gracias a una de sus principales características "la trazabilidad," ofrece la posibilidad de garantizar el origen de los alimentos, así como realizar un rastreo desde que el alimento es producido en la granja hasta su llegada a la mesa del consumidor mediante un código QR y, además, puede aplicarse a campos como el turismo culinario, el agroturismo o enoturismo.

IBM, el gigante tecnológico ha empezado a utilizar esta tecnología en empresas como Nestlé o los supermercados Walmart para priorizar la salud de los consumidores. HAZI, la entidad que certifica y promociona Productos de Calidad Vascos de la marca Euskolabel, también está utilizando blockchain para garantizar la cadena de seguridad en productos bovinos producidos, transformados y/o elaborados en la Comunidad Autónoma del País Vasco.

Por otro lado, el etiquetado de productos ha sido utilizado como una táctica de marketing durante mucho tiempo en la industria de la restauración, pues los establecimientos tratan de hacer más atractivos sus productos, a veces incluso más de lo que realmente son. Finalmente es el consumidor quien debe valorar la autenticidad y exactitud de dicho etiquetado, y en numerosos casos esto no puede hacerse a priori, pues primero se debe comprar el producto o servicio y hasta que no es obtenido no se puede hacer comparativa. Esto deja al cliente potencialmente descubierto frente a la exageración o el aumento desmesurado de expectativas causadas por un marketing abusivo. Con el uso de blockchain se aportará claridad y transparencia, haciendo así que la duda sobre estos productos sea resuelta. Como ejemplo principal se presenta a la empresa Where Foods come From, en Colorado, que trabaja mediante blockchain verificando y certificando comida en la industria turística (Willie, 2019).

También se han desarrollado proyectos de blockchain en el enoturismo. VinAssure es una plataforma creada para la industria vinícola que ofrece una forma más inteligente y segura de rastrear los vinos desde el viñedo hasta el consumidor final. VinAssure se ejecuta con IBM Cloud y se basa en IBM Blockchain Transparent Supply. Está diseñado para utilizar 
tecnologías avanzadas que incluyen blockchain, IA y nube con tal de optimizar resultados.

\section{Finanzas}

Blockchain, gracias a su descentralización, simplifica los pagos, haciéndolos más rápidos, seguros, efectivos y trazables, pues todas las transacciones que se realizan a través de la red de blockchain quedan registradas en la cadena de bloques y no se pueden modificar, y además al ser un sistema descentralizado no habría intermediarios que pudiesen intervenir o rastrear el pago en cuestión.

Del mismo modo, ya no sería necesario hacer cambios de divisas al viajar a otro país o quedar sujetos a la fiabilidad o volatilidad de estas. Es por ello por lo que realizar pagos a través de esta tecnología puede suponer una diferencia más que relevante en lo referente a transacciones overseas (extranjeras o exteriores).

\section{Turista}

La tecnología blockchain también puede aportar ciertos beneficios al consumidor final. El viajero actual utiliza mucho las reseñas de usuarios en el momento de escoger un destino; sin embargo, no siempre se puede garantizar quien lo escribió con exactitud, mediante blockchain toda la información que aparece en la red es pública, confiable y segura, logrando una mayor transparencia y aumentado la confianza del consumidor. Esta tecnología se ha venido convirtiendo de una forma paulatina en una herramienta que promete revolucionar los programas de lealtad para el cliente.

Un pilar fundamental de blockchain es el aspecto de la identidad digital auto soberana. Básicamente, cada ser humano será portador de su propia información, la cual facilitará con una finalidad concreta por un tiempo determinado. En ningún momento se acumulará un dato, en propiedad de un tercero.

Estos datos se pueden subdividir en categorías, y así poder ofrecerlas según quién y para qué la solicita. Dándole, además, una característica que hasta hoy no es posible, que es concretar durante cuánto tiempo y para qué se va a usar ese dato e incluso quién puede llegar a verlo.

La blockchain también podría ser beneficiosa para los seguros de empresas de alquiler de vehículos. Tradicionalmente, estos seguros se han basado en características del conductor como la edad, información personal, historial de conducción, etc. A través de esta tecnología las compañías serían capaces de incorporar más información del comportamiento del conductor, como la velocidad a la que circula o si se realizan frenadas fuertes. Además, mediante smart contracts se podría medir el tiempo que el usuario 
conduce al día para ofrecer pólizas de seguros que ajusten el precio en función del tiempo de conducción realizado (Rugg, 2019).

\section{Blockchain y el turismo}

El blockchain en el turismo se encuentra es una fase muy incipiente a pesar de las expectativas puestas debido al impacto que causa en el sector. Estudios realizados muestran que tecnología tendrá una expansión en los siguientes lustros enfocada en 12 países: China encabezando la lista, Estados Unidos, Alemania, Japón, Reino Unido, India, Francia, Emiratos Árabes Unidos, Suecia, Luxemburgo, España e Italia; estimando un aumento monetario con valor al 1,4\% del PIB mundial (Instituto Nacional de Contadores Públicos [INCP], 2020).

Mena (2021) plantea que, Estados Unidos sin aun tener las criptodivisas como monedas de curso legal, es el país con la red más extendida de cajeros automáticos que operan con criptomonedas, con más de 20 mil máquinas disponibles en su territorio. Mientras que, en el continente europeo, la lista la encabeza Reino Unido con 159, seguido de España y Austria.

\section{Figura 4}

Principales países con cajeros automáticos de criptomonedas

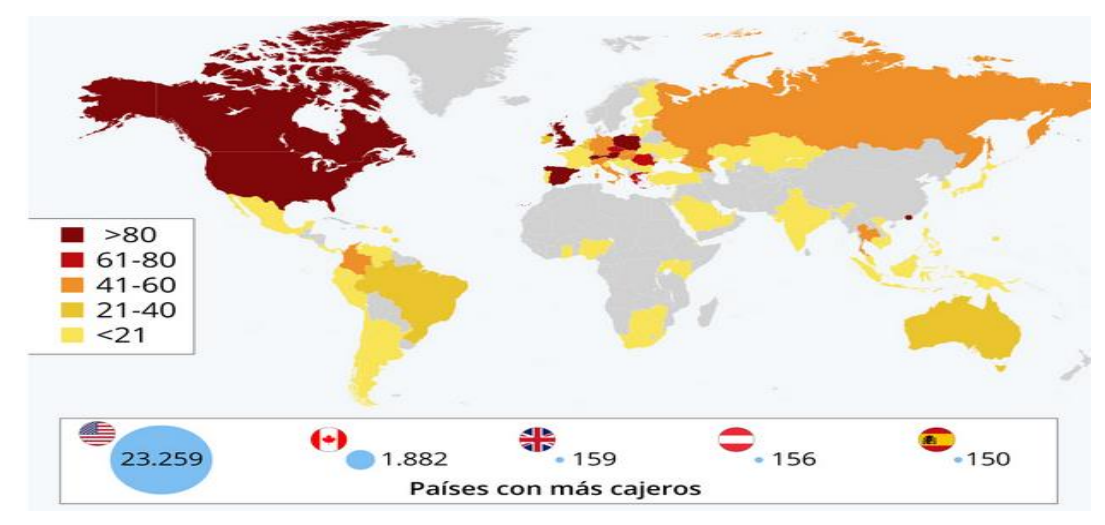

Fuente: Mena (2021)

Por otra parte, desde el punto de vista nacional (Mesa, 2020), el uso de criptoactivos monetarios parte de principales mercados emisores turísticos cubanos hace que estas naciones se ubiquen en los principales tres bloques de países activos en la adopción de criptomonedas. 


\section{Tabla 1}

Principales mercados emisores turísticos cubano

\begin{tabular}{lrr}
\hline Países visitantes & Año 2019 & Año 2020 \\
\hline Canadá & 159022 & 162307 \\
Comunidad Cubana en el Exterior & 42945 & 37394 \\
Federación Rusa & 16742 & 24842 \\
Estados Unidos de América & 62416 & 19464 \\
Francia & 22354 & 16871 \\
Alemania & 20891 & 14679 \\
Italia & 16522 & 13129 \\
México & 10951 & 10580 \\
España & 9485 & 8910 \\
Inglaterra & 15945 & 7898 \\
Otros & 112345 & 77597 \\
\hline \multicolumn{1}{c}{ Fus }
\end{tabular}

Fuente: Elaboración propia, datos tomados de ONEI (2021)

\section{Tabla 2}

Países activos en la adopción de criptomonedas

\begin{tabular}{|c|c|}
\hline Bloques & Países \\
\hline \multirow{5}{*}{ Primer Bloque } & Turquía \\
\hline & Brasil \\
\hline & Colombia \\
\hline & Argentina \\
\hline & Sudáfrica \\
\hline \multirow{6}{*}{ Segundo Bloque } & México \\
\hline & Chile \\
\hline & China \\
\hline & Indonesia \\
\hline & España \\
\hline & Rusia \\
\hline \multirow{7}{*}{ Tercer Bloque } & Dinamarca \\
\hline & Australia \\
\hline & Reino Unido \\
\hline & Estados Unidos \\
\hline & Francia \\
\hline & Alemania \\
\hline & Japón. \\
\hline
\end{tabular}




\section{Regulaciones en Cuba}

El comercio electrónico en Cuba carece de normativa especial, más allá de ciertas iniciativas estatales de realizar pruebas piloto sobre este. Al respecto, el Acuerdo del Consejo de Ministros por el que se aprobaron los lineamientos para el Comercio Electrónico del 26 de diciembre de 2005 establece que, dentro de esta prueba, los Ministerios del Interior y de Justicia tenían la obligación de proponer o dictar todas las normas necesarias para darle validez legal a los actos celebrados en forma electrónica.

A su vez, la Ley N. 7 del 19 de agosto de 1977 (la "Ley Cubana de Procedimiento Civil, Administrativo y Laboral"), en su reforma de 2006, introdujo el artículo 777, relativo a los procesos de índole económica, que establece que las pruebas consisten en documentos, comprendidos los electrónicos o digitales, dictámenes de peritos, reconocimiento judicial, y declaraciones de testigos o especialistas, y demás medios que se reconocen y regulan en esta Ley.

Validez de las firmas digitales

El Reglamento Cubano sobre el funcionamiento de la infraestructura de llave pública no establece que las firmas digitales son equivalentes a las firmas manuscritas. Siguiendo a la doctrina en la materia mencionada antes, en la medida que sea de aplicación el principio de libertad de formas, será posible considerar equiparable una firma digital a una firma manuscrita.

\section{Regulación sobre contratos inteligentes}

No existe regulación sobre contratos inteligentes en Cuba. Al no existir una regulación especial ni una prohibición al respecto, si se reúnen los requisitos legales para tener un contrato mediante la forma de un contrato inteligente, no debería haber obstáculos para que el contrato inteligente sea considerado legal.

\section{Retos más relevantes de la economía y las finanzas cubanas para utilizar Blockchain}

Los principales retos son:

$\checkmark$ Contar con divisas y sistemas de pagos soberanos y seguros que defiendan del acoso y la persecución financiera.

$\checkmark$ Acceso a financiamiento. Captación de fondos para la economía general y para financiar proyectos concretos, no vulnerables al acoso por parte de Estados Unidos.

$\checkmark$ La imposibilidad del uso del USD como moneda de cobro y pago del comercio exterior que obliga al empleo de otras divisas lo que expone a las finanzas del país a un excesivo riesgo cambiario

$\checkmark$ Bajo conocimiento por parte de las MIPYMES de los beneficios que podría aportar esta tecnología. 
Las criptomonedas, en su relativamente corta existencia, han exhibido algunas características que pudieran resultar convenientes para Cuba, en su objetivo de poseer mecanismos de pago protegidos que permitan cobrar las exportaciones, pagar las importaciones y canalizar flujos comerciales y de crédito.

Cuba enfrenta el reto de tener prácticamente cerrada la red interbancaria mundial, lo cual se traduce en pocos bancos dispuestos a tener cuentas de entidades cubanas y a prestar servicios elementales de cuenta, de cobros y pagos corrientes y de servicios comerciales, como apertura, confirmación, aviso y pago de Cartas de Créditos. Esta restricción sensible abarca no solo cuentas en USD o bancos parcial o totalmente propiedad de empresas norteamericanas, sino que se extiende a prácticamente cualquier banco y moneda. Las sanciones a decenas de bancos e instituciones financieras europeas, latinoamericanas y asiáticas por tener relaciones con Cuba son muestra de ello.

La capacidad demostrada por la tecnología de cadena de bloques, así como las experiencias exitosas existentes como el protocolo Ethereum y Ripple, en el diseño y explotación de plataformas de pago eficientes, baratas y seguras; hace concluir que resultaría muy oportuno explorar la viabilidad de crear ecosistemas, con implicación de diferentes actores internacionales, que permitan, por ejemplo, contar con esquemas de venta y cobro de servicios turísticos cubanos por parte de agencias en todo el mundo. Pudieran crearse diferentes ecosistemas independientes, o uno general que actúe como pasarela de pago para todos estos servicios y, al mismo tiempo, brinde acceso a financiamientos vinculados a proyectos concretos o a entidades.

\section{Conclusiones}

- El surgimiento de las criptomonedas, lideradas por el bitcoin como principal, obedece a la existencia de condiciones objetivas y subjetivas favorables pasa su implementación en el sector turístico.

- El blockchain desde su aparición se ha convertido en una verdadera revolución tecnológica que ha influido en la manera en que funcionan las finanzas a niveles internacionales, su uso ha rebasado la esfera financiera para impactar con fuerza la vida cotidiana en el orden social, económico y cultural de muchos países.

- Blockchain aún se enfrenta a una serie de desafíos técnicos, normativos y prácticos que limitan la posibilidad de capitalizar íntegramente todas las bondades que ofrece.

- Se debe integrar la tecnología blockchain de forma transversal en la sociedad para que la transformación digital del sector turístico sea una realidad firme y puedan desarrollarse proyectos de gran impacto a largo plazo.

- Actualmente es relativamente difícil de decir si la tecnología blockchain en Cuba cumplirá con todas las expectativas que se han depositado para transformarse en un instrumento fundamental para el desarrollo y la inclusión financiera. Lo que 
está claro es que tiene el potencial de añadir valor si su aplicación se lleva a cabo de manera estratégica, creativa y responsable.

\section{Referencias bibliográficas}

Allende L., M. (2018). Blockchain. Cómo desarrollar confianza en entornos complejos para generar valor de impacto social.

Berenguer, J. (2017). Blockchain la tecnología que está en boca de todos. Andbank.

Binance Academy. (12 de diciembre de 2018). Ventajas y desventajas del Blockchain. Binance Academy: https://www.google.com/amp/s/academy.binance.com/es/articles/positives-andnegatives-of-blockchain.amp

Castro, J. (17 de abril de 2021). ¿Cuántas criptomonedas existen en el mundo además del Bitcoin? La República.

Coin ATM Radar. (2021). Coin ATM Radar: https://coinatmradar.com/

El Economista. (12 de abril de 2021). El bitcoin vuelve a acercarse a su máximo histórico justo antes de la salida a bolsa de la platafotma Coinbase. El Economista. Obtenido de https://www.eleconomista.es/mercadoscotizaciones/amp/11153276/El-bitcoin-vuelve-a-acercarse-a-su-maximohistorico-justo-antes-de-la-salida-a-bolsa-de-la-plataforma-Coinbase

Equipo Cazoo. (mayo de 2021). ¿Qué es una red Trustless, sin necesidad de confianza? Obtenido de Cazoo: https://www.google.com/amp/s/cazoo.it/impare-lecriptovalute/cose-una-rete-trustless-senza-il-biosogno-della-fiducia/amp/

Fernández, R. (11 de diciembre de 2020). Número de transacciones por día en Bitcoins 2015-2020. Statista: https://es.statista.com/estadisticas/549090/numero-detransacciones-por-dia-en-bitcoins/

Ganne, E. (2018). ¿Can Blockchain revolutionize international trade? Geneva: World Trade Organization.

Iglesias F., A. (03 de junio de 2018). La historia del blockchain en cinco hitosde 1997 a hoy. Obtenido de Hoy: https://computerhoy.com/reportajes/industria/historia-blockchain-cinco-hitos1997-hoy-257817 
Instituto Nacional de Contadores Públicos [INCP]. (16 de octubre de 2020). 12 países que liderarán el blockchain mundial. https://incp.org.co/12-paises-que-lideraranel-blockchain-mundial/

Kwok, A., \& Koh, S. (26 de agosto de 2018). Is blockchain technology a watershed for tourism development? (T. \&. Group, Ed.) Current Issues in Tourism, VOL. 22(20), págs. 2447-2452. doi:10.1080/13683500.2018.1513460

López B., D. I. (4 de febrero de 2019). Conoce la historia del blockchain y su evolución. Obtenido de Solution Times: https://www.solutiontimes.news/2019/02/04/historia-del-blockchain-evolucion/

Mena, M. (08 de septiembre de 2021). ¿Qué países tienen más cajeros de criptomonedas? Obtenido de Statista: https://es.statista.com/grafico/25725/numero-de-cajeros-debitcoin-y-otras-criptomonedas-en-2021-por-pais/

Mesa, R. (30 de noviembre de 2020). Descubre los países más activos en el uso de criptomonedas. Directivos y Empresas: https://www.directivosyempresas.com/noticias/macroeconomia/descubre-lospaises-mas-activos-en-el-uso-de-criptomonedas/

ONEI. (14 de mayo de 2021). Turismo Nacional e Internacional. Indicadores seleccionados. Enero-diciembre 2020. Obtenido de ONEI: http://www.onei.gob.cu/node/15651

Palomo-Zurdo, R. (12 de junio de 2018). "Blockchain": la desentralización del poder y su aplicación en la defensa. Obtenido de Instituto Español de Estudios Estratégicos: https://www.ieee.es

Rugg, R. (2019). Blockchain for insurance in 2019. Obtenido de International Travel \& Health Insurance Journal.: https://www.itij.com/latest/long-read/blockchaininsurance-2019

Vector ITC. (2018). Blockchain: disrupción, valor y seguridad. Madrid. doi: https://www.vectoritcgroup.com

Willie, P. (2019). ¿Can all sectors of the hospitality and tourism industry be influenced by the innovation of Blockchain technology? Worldwide Hospitality and Tourism Themes, 112-120.

Yahari Navarro, B. (2019). Blockchain y sus aplicaciones. Universidad Católica Nuestra Señora de la Asunción, Asuncion, Paraguay. Obtenido de Universidad Católica: http://www.universidadcatolica.edu.py/ 


\section{EPDigital}

El artículo que se publica es de exclusiva responsabilidad de los autores y no necesariamente reflejan el pensamiento de la Revista Explorador Digital.

\section{\Cigncia}

El artículo queda en propiedad de la revista y, por tanto, su publicación parcial y/o total en otro medio tiene que ser autorizado por el director de la Revista Explorador Digital.
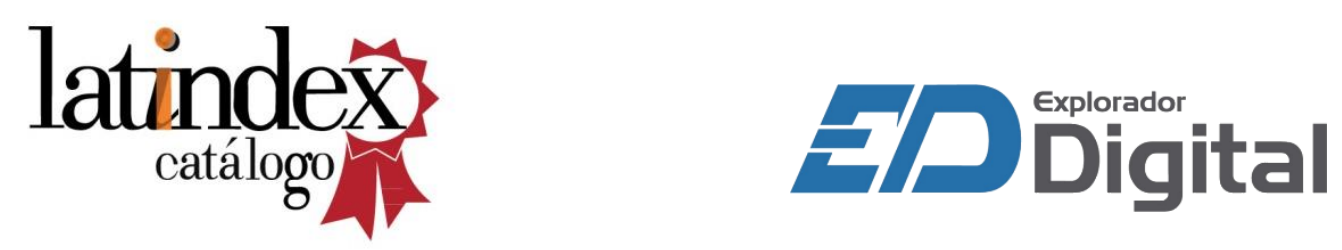

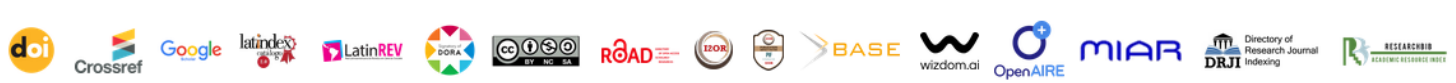 ERRाHDUS pCiteractor

